\title{
Hacker statt Cracker
}

Liebe Leserin, lieber Leser,

Hacker gelangten jüngst in den USA über das Infotainmentsystem eines Jeep in den CAN-Bus und steuerten das Fahrzeug fern. Oder waren es Cracker und nicht Hacker? Die Bezeichnung Hacken ist fälschlicherweise in der Öffentlichkeit in Verruf geraten - gleichbedeutend mit Computerkriminalität. Die Kriminellen sind aber Cracker. Sie stehlen OnlineIdentitäten und nutzen Schwachstellen in Systemen aus. Cracker setzen HackerWissen böswillig ein.

Gut hingegen meinten es die beiden Jeep-Hacker Charlie Miller und Chris Valasek. Sie deckten nämlich in einem abgesicherten Test Sicherheitslücken auf. Zunächst verstellten sie die Klimaanlage und den Radiosender. Schließlich übernahmen sie die Kontrolle über das Gaspedal und bremsten das Fahrzeug bei voller Fahrt ab. Der Fahrer konnte trotz manueller Gegenmaßnahmen diese Eingriffe weder verhindern noch stoppen.

Gut meint es auch Prof. Dr.-Ing. Christof Paar, Kryptografie-Experte und Inhaber des Lehrstuhls für Eingebettete Sicherheit an der Ruhr-Universität Bochum. „Wir müssen versuchen, die Systeme mit einem gewissen Killerinstinkt zu hacken. Die Automobilisten sind nicht darauf eingestellt, und ich rate in diesem Zusammenhang dringend zu Penetrationstest im Feld.“ Man muss sich also sprichwörtlich in die Rolle des Feindes versetzen. „Das ist eine hochwissenschaftliche Aufgabe, die niemals enden wird, da sich die Methoden der Angreifer ständig verbessern und anpassen“, betont Paar.

Vermehrt warnen weitere Experten vor Sicherheitslücken, allerdings auch in der Hardware. Die Branche konzentriere sich aber zu sehr auf die Softwaresicherheit. „Ein großes Forschungsthema ist beispielsweise das Design von HardwareTrojanern. Hier geht es darum, ob wir
Systeme schwächen können, indem wir die Hardware manipulieren“, erklärt Paar. Das sei ein neuer Ansatz, nachdem man sich bisher lediglich mit dem Suchen und Auffinden von Manipulationen beschäftigt habe.

Für Sicherheitsexperten ist das eine sehr unschöne Situation, denn man kann Hardware, beispielsweise eine CPU, auf der untersten Ebene manipulieren, entweder beim Herstellungsprozess oder aber bereits beim Design. „Es gibt allerdings erschreckend wenige Fachleute, die wissen, wie solche Trojaner überhaupt aussehen“, stellt Paar fest.

Was bedeutet das für die Ingenieure in der Automobilindustrie? Ihnen könnte man zumindest ein Security-Team zur Seite stellen, das ihnen über die Schulter guckt und ihnen zeigt, wie ein Cracker das System angreifen und zerlegen könnte. So wie das Miller und Valasek getan haben. In Ansätzen wird dies heute bereits praktiziert, es sollte aber institutionalisiert werden.

Herzliche Grüße

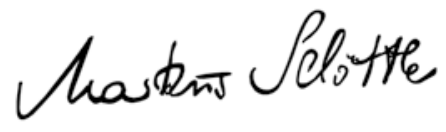

Markus Schöttle

Stellvertretender Chefredakteur

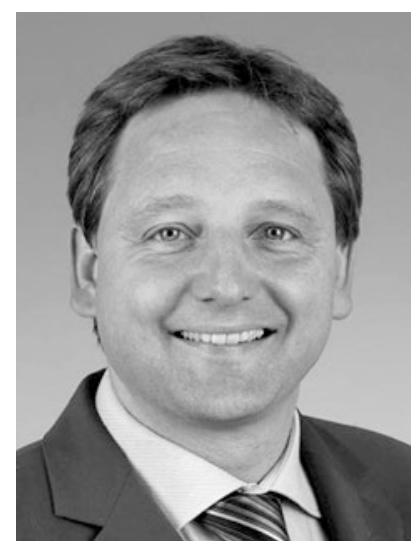

